\title{
MANDİBULA POSTERİORUNDA BÜYÜK BOYUTLU KOMPLEKS ODONTOM: VAKA RAPORU
}

\section{LARGE COMPLEX ODONTOMA IN POSTERIOR MANDIBLE: REPORT OF A CASE}

\author{
Doç. Dr. Mehmet Melih ÖMEZLİ \\ Dr. Öğr. Üyesi Damla TORUL*
}

Doç. Dr. Ferhat AYRANCI ${ }^{*}$

Arş. Gör. Dt. Kadircan KAHVECI ${ }^{*}$

Arş. Gör. Dt. Hasan AKPINAR*

Makale Kodu/Article code: 4115

Makale Gönderilme tarihi: 12.07.2019

Kabul Tarihi: 31.10 .2019

DOI : $10.17567 /$ ataunidfd. 640370
M. Melih Ömezli : ORCID ID: 0000-0002-6606-6593

Ferhat AyrancI: ORCID ID: 0000-0001-7126-5696

Damla Torul: ORCID ID: 0000-0003-2323-606x

Kadircan Kahveci: ORCID ID: 0000-0001-8532-3367

Hasan Akpınar: ORCID ID: 0000-0001-5304-3897

\section{öz}

Odontomlar mine, sement ve dentin gibi dișe ait yapılar içeren benign tümörlerdir. En sık 2. dekatta gözlenen odontomların prevalansı cinsiyetler arasında farklılık göstermemektedir. Histolojik olarak kompleks ve kompound olmak üzere iki tip olan odontomlardan kompound tip maksilla anteriorda, kompleks tip ise posterior bölgelerde daha sık görülmektedir. Genellikle asemptomatik olan odontomlar; enfeksiyon, kemik ekspansiyonu, kistik değişim ve erüpsiyon problemleri gibi patolojik durumlara sebebiyet verebilmektedir. Odontomların neden olduğu erüpsiyon problemleri tümörün eksizyonunu takiben spontan olarak düzelebilmektedir. Spontan erüpsiyonun sağlanamadığı vakalarda ortodontik tedavi gibi ek bir yaklaşım gerekliliği ortaya çıabilmektedir.

Bu vaka raporunda, mandibula posterior bölgede lokalize büyük boyutlu kompleks odontomun tedavisi ve odontom nedeniyle gömülü kalan daimi sol mandibular birinci molar dişin takibi sunulmaktadır.

Anahtar Kelimeler: Kompleks odontom, mandibula, eksizyon

\section{ABSTRACT}

Odontomas are benign tumors that contain dental structures such as enamel, cementum and dentin. The prevalence of odontomas that are most commonly observed in the second decade, does not differ between genders. Histologically, there are two types of odontoma as complex and compound; the compound type seen maxillary anterior region more commonly while the complex type is more common in the posterior regions. Odontomas, which are generally asymptomatic, can cause pathological conditions such as infection, bone expansion, cystic change, and eruption problems. Eruption problems caused by odontomas can resolve spontaneously following excision of the tumor. In cases where spontaneous eruption cannot be achieved, an additional approach, such as orthodontic treatment, should be considered.

In this case report, treatment of large complex odontoma localized in the posterior mandible, and follow-up of the permanent first molar tooth which fail to erupt due to odontoma are presented.

Keywords: Complex odontoma, mandible, excision

* Ordu Üniversitesi, Diș Hekimliği Fakültesi, Ağız, Diș ve Çene Cerrahisi Anabilim Dalı, Ordu

Kaynakça Bilgisi: Ömezli MM, Ayrancı F, Torul D, Kahveci K, Akpınar H. Mandibula posteriorunda büyük boyutlu kompleks odontom: vaka raporu. Atatürk Üniv Diş Hek Fak Derg 2020; 30: 650-3.

Citation Information: Omezli MM, Ayranci F, Torul D, Kahveci K, Akpinar H. Large complex odontoma in posterior mandible: report of a case. $J$ Dent Fac Atatürk Uni 2020; 30: 650-3.

\section{GİRİs}

Odontom maksillofasiyal bölgede en sık görülen, mine, sement ve dentin gibi dişe ait yapılar içeren benign bir odontojenik tümördür. ${ }^{1}$ Sınırlı ve yavaş büyüme özelliğinden dolayı odontomlar hamartom olarak da adlandırılmaktadır. ${ }^{2}$ Literatürde 6-
77 yaş gibi geniş bir aralıkta görülebildikleri bildirilse de bu lezyonlar en sık 2. dekatta gözlenmektedir. ${ }^{3}$ Cinsiyet açısından ise odontomların gözlenme sıklığı kadın ve erkeklerde farklılık göstermemektedir. ${ }^{4}$ Literatürde bildirilen birçok vakada odontomun büyüklüğü 3 cm'yi geçmemektedir. ${ }^{5} 3$ cm'den büyük olan odontomlar dev odontom olarak isimlendirilmektedir. ${ }^{2,6}$ 
Odontomlar histolojik olarak irregüler yapılardan oluşan kompleks ve diş benzeri yapılardan oluşan kompound olmak üzere iki tiptir. ${ }^{7}$ Kompound odontomlar maksilla anteriorda daha sık gözlenirken kompleks odontomlar çenelerin posteriorunda daha sık görülmektedir. ${ }^{3}$ Radyolojik olarak odontomlar radyolüsent kapsül ile çevrili, düzensiz kortikal kemik içeren radyopasitelerdir ve genellikle tesadüfen teşhis edilirler. ${ }^{8}$ Odontomlar genellikle asemptomatik klinik davranış sergilerler; ancak bazı vakalarda kemik ekspansiyonu, enfeksiyon, erüpsiyon problemleri, maloklüzyon, devitalizasyon, diş ve kemiklerde rezorbsiyon, ve kistik değişime neden olabilecekleri bildirilmiştir. ${ }^{5,9,10}$ Odontomların tedavileri eksizyondur. ${ }^{11}$ Lezyon büyüklüğüne göre farklı yaklaşımlar kullanılabilmektedir. ${ }^{1,12}$

$\mathrm{Bu}$ vaka raporunda, daimi sol mandibular 1. molar dişin sürmesini engelleyen dev bir odontomun eksizyonu ve 2 yıllık takipte daimi molar dişin fizyolojik erüpsiyonu sunulmuştur.

\section{OLGU SUNUMU}

Kliniğimize başvuran 9 yaşındaki erkek hastanın sol mandibular posterior bölgesinde ağrısız hafif bir ekspansiyon tespit edildi. Alınan panoramik radyografide angulusa kadar uzanan, daimi sol mandibular 1. molar dişi içeren, iyi sınırlı, radyopak ve etrafı radyolüsent hale ile çevrili lezyon tespit edildi (Şekil 1). Lezyonun anatomik yapılara yakınlığı ve boyutu nedeniyle Dental Volumetrik Tomografi (DVT) alındı. Aksiyel kesit ve 3D DVT görüntülerinde lezyo- nun inferior alveolar sinire yakın komşulukta olduğu, bukkal ve lingual korteksleri farklı seviyelerde perfore ettiği belirlendi (Şekil 2, 3). Boyutları 3,5*2,5*1,5 cm olan kitlenin genel anestezi altında eksizyonu planlandı (Şekil 4).

Hastanın yasal temsilcisinden aydınlatılmış onam alındı. Genel anestezi altında lokal anestezi desteği ile alt sol kanin dişten ramusa uzanan tam kalınlıkta mukoperiosteal flep kaldırıldı. Frezler yardımıyla lezyon bölgesine ulaşıldı. Bukkal ve lingual kortekste fraktür oluşumunu engellemek için lezyon parçalara ayrıldı. Lezyona anatomik yakınlık gösteren inferior alveolar sinir ve daimi sol mandibular 1. molar dişe zarar vermemeye özen gösterildi. Ardından flep primer olarak kapatıldı. Hastaya 1 hafta süreyle antibiyotik, analjezik ve gargara verildi. Klinik kontrollerde lezyon bölgesinin sorunsuz iyileştiği ve herhangi bir komplikasyonun gelişmediği gözlendi. Spesimenin histopatolojik olarak incelenmesi sonucu kesin tanı kompleks odontom olarak rapor edildi. Hastanın 2 yıllık takibinde alınan radyografide lezyon bölgesinde yeni kemik olu- şumu ve daimi sol mandibular 1. molar dişin fizyolojik olarak sürmeye başladığı gözlendi (Şekil 5).

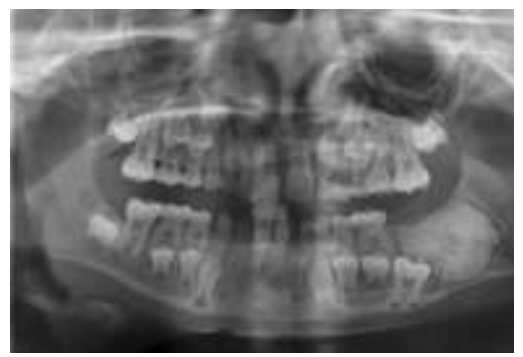

Şekil 1. Preoperatif radyografi

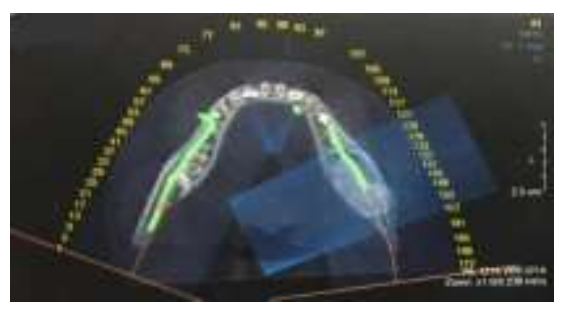

Şekil 2. Aksiyal kesit DVT

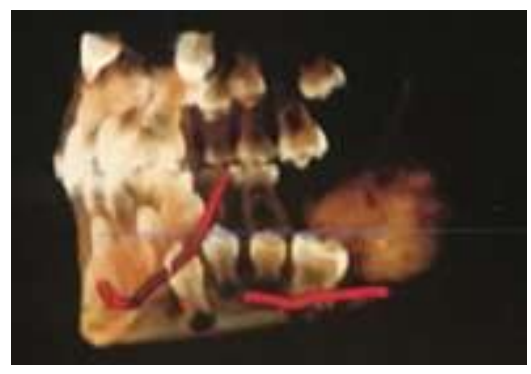

Şekil 3. Lezyonun 3D görünümü

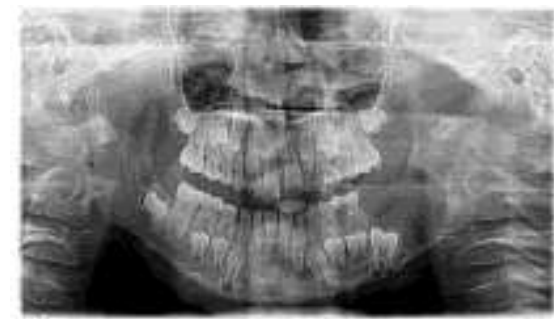

Şekil 4. Cerrahi sonrası radyografi

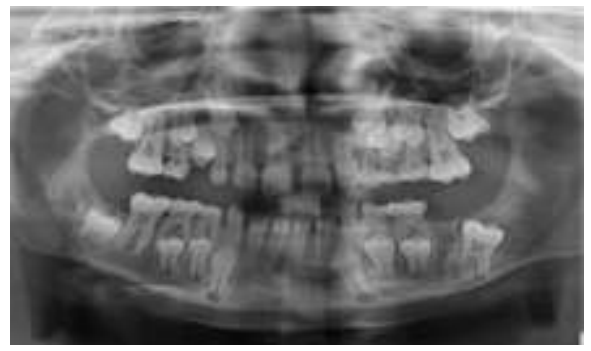

Şekil 5. Postoperatif radyografi 


\section{TARTIŞMA}

Odontom agresif büyüme göstermeyen, odontojenik dokulardan köken alan benign bir tümördür. ${ }^{13}$ Maksillofasiyal bölgede görülen odontojenik tümörlerin $\% 41,8^{\prime}$ ini odontomlar oluşturur. ${ }^{1}$ Odontomların etiyolojisi tam olarak bilinmemektedir. Ancak; primer dentisyon sürecindeki enfeksiyon ve enflamasyona eşlik eden travma, heredite, genetik mutasyon, ile Gardner, Hermann ve Bazall Hücreli Nevus sendromları gibi predispozan faktörlerin epiteliyal ve mezenşimal hücrelerin proliferasyonunu indükleyerek odontom oluşumuna zemin hazırladığı düşünülmektedir. 4, 9, 14

Odontomlar genel olarak asemptomatiktir. Ancak literatürde semptomatik hale gelerek teşhis edilen vakalar da bulunmaktadır. Akerzoul ve arkadaşlarının rapor ettiği dev odontom vakasında ekspansiyon gözlenmiştir. ${ }^{13}$ Park ve arkadaşlarının angulus bölgesinde teşhis ettiği diğer bir olgu raporunda ekspansiyona ek olarak hastanın ağrı şikayeti olduğunu bildirilmiştir. ${ }^{2}$ Diğer yandan ekspoze olabilme potansiyeli göstermesi de bu tümörlerin klinik olarak teşhis edilmesine neden olabilmektedir. Raval ve arkadaşları maksilla anteriorda ekspoze odontomlar rapor etmiştir. ${ }^{15}$ Perumal ve arkadaşlarının vaka raporunda ekstraoral olarak ekspoze olmuş dev odontom rapor edilmiştir. ${ }^{16}$ Agrawal ve arkadaşları da ekstraoral olarak ekspoze olmuş bir odontom olgusu bildirmişlerdir. ${ }^{17} \mathrm{Bu}$ vaka raporunda da benzer şekilde mandibula posteriorunda lokalize, ağıısız, hafif ekspansiyon yapmış dev bir odontom tespit edilmiştir.

Odontomlar genellikle panoramik radyografi ile teşhis edilirler. ${ }^{14}$ Bununla birlikte tümörün çok büyük boyutlara ulaştı̆ı̆ ve anatomik yapılara yakınlık gösterdiği durumlarda DVT gibi daha ileri görüntüleme tekniklerine de ihtiyaç duyulabilmektedir. ${ }^{8,18}$ Büyüklüğü $3 \mathrm{~cm}$ 'den fazla olan odontomlar dev odontom veya büyük odontom olarak adlandırımaktadır. ${ }^{2}$ Literatürde lezyon büyüklüğü $3 \mathrm{~cm}$ 'den $8 \mathrm{~cm}$ 'ye kadar değişen vakalar bildirilmiştir. ${ }^{1-3,6,8,10,12,13,16,17,19,20}$ (Tablo 1). Dev odontom olarak tanımlanabilecek boyutlarda olan bizim vakamızda da tümör hacmi ve anatomik yapılara yakınlık nedeniyle panoramik inceleme yanında DVT incelemesinin de yapılması gerekli görülmüştür.

Odontomlar semptom vermedikçe takibini öneren araştırmacılar bulunsa da genel görüş odontomların eksize edilmesi yönündedir. ${ }^{6}$ Büyük boyuttaki odontomların eksizyonu için farklı yaklaşımlar literatürde bildirilmiştir. ${ }^{19}$ Bu yaklaşımların seçiminde lezyon büyüklüğü primer faktördür. ${ }^{1}$ Christopher ve arkadaş- larının vaka raporunda büyük boyutlu bir odontomun eksizyonu için sagittal split osteoteomisi kullanılmıştır. ${ }^{12}$ Başka bir vaka raporunda Park ve arkadaşları angulus bölgesindeki odontomun eksizyonu ile birlikte iliak greft ve mini plaklarla defekt bölgesini rekonstrükte etmişlerdir. ${ }^{2}$ Cezairli ve arkadaşlarının vaka raporunda ise büyük boyutlu odontomun tek seferde eksizyonu tamamlanmış ve fraktür riskine karşı ortodontik elastikler kullanımıştır. ${ }^{1}$ Bizim vakamızda da lokal eksizyon uygulanmış olup kalan sağlıklı kemik miktarı değerlendirilerek ek bir önleme ihtiyaç duyulmamıştır.

Tablo 1. Literatürdeki dev odontom vakaları ve tedavi yaklaşımları

\begin{tabular}{|c|c|c|c|c|}
\hline Yazar/yıl & $\begin{array}{l}\text { Lezyon } \\
\text { Yerleşimi }\end{array}$ & $\begin{array}{c}\text { Lezyon } \\
\text { Büyüklüğü }\end{array}$ & $\begin{array}{c}\text { Cerrahi } \\
\text { Yaklaşım }\end{array}$ & Ek Önlem \\
\hline Casap/2006 19 & $\begin{array}{l}\text { Mandibula } \\
\text { posterior }\end{array}$ & $\#$ & IO/SSO & Mini plak \\
\hline Perumal/2009 & $\begin{array}{l}\text { Mandibula } \\
\text { posterior }\end{array}$ & $\begin{array}{c}5.5 * 4 * 2.5 \\
\mathrm{~cm}\end{array}$ & IO/E & - \\
\hline Utumi/2011 ${ }^{8}$ & $\begin{array}{l}\text { Maksilla } \\
\text { anterior }\end{array}$ & $\begin{array}{c}4 \mathrm{~cm} \\
\text { çapında }\end{array}$ & IO/E & $\begin{array}{c}\text { İliak greft } \\
+ \text { titanyum } \\
\text { mesh }\end{array}$ \\
\hline Carvalho/2011 10 & $\begin{array}{l}\text { Maksilla } \\
\text { posterior }\end{array}$ & $\begin{array}{c}7 \mathrm{~cm} \\
\text { capında }\end{array}$ & IO/E & - \\
\hline Agrawal/2012 17 & $\begin{array}{l}\text { Mandibula } \\
\text { posterior }\end{array}$ & \# & IO/E & - \\
\hline Lehman $/ 2013^{3}$ & $\begin{array}{l}\text { Mandibula } \\
\text { posterior }\end{array}$ & $8 \mathrm{~cm} * 4 \mathrm{~cm}$ & IO/E & Arch bar \\
\hline Visioli/2015 20 & $\begin{array}{l}\text { Maksiller } \\
\text { sinus }\end{array}$ & \# & IO/E & - \\
\hline Spini/2015 & $\begin{array}{l}\text { Mandibula } \\
\text { anterior }\end{array}$ & $\begin{array}{c}6 \mathrm{~cm} \\
\text { çapında }\end{array}$ & IO/E & - \\
\hline Akerzoul/2016 ${ }^{13}$ & $\begin{array}{l}\text { Mandibula } \\
\text { posterior }\end{array}$ & $6 * 6 \mathrm{~cm}$ & IO/E & - \\
\hline Cezairli/2017 ${ }^{1}$ & $\begin{array}{l}\text { Mandibula } \\
\text { posterior }\end{array}$ & $3.5 * 3 * 2 \mathrm{~cm}$ & IO/E & $\begin{array}{l}\text { Ortodontik } \\
\text { elastik }\end{array}$ \\
\hline Christopher $/ 2017^{12}$ & $\begin{array}{l}\text { Mandibula } \\
\text { posterior }\end{array}$ & \# & IO/SSO & Mini plak \\
\hline Park $/ 2018^{2}$ & $\begin{array}{l}\text { Mandibula } \\
\text { posterior }\end{array}$ & $3 * 2.5 * 2 \mathrm{~cm}$ & IO/E & $\begin{array}{l}\text { İliak greft } \\
+ \text { mini plak }\end{array}$ \\
\hline
\end{tabular}

IO: Intraoral yaklaşım SSO: Sagittal Split Osteotomisi E: Konvansiyonel Eksizyon \#: Lezyon büyüklüğ̈̈̈ bilgisi bulunmamaktadır.

Sonuç olarak odontomlar çenelerde erüpsiyon problemleri gibi birçok probleme neden olabilmektedir. Bizim vakamızda daimi molar dişin erüpsiyonunu engelleyen odontomun eksizyonunun ardından ek ortodontik önlem gerekmeksizin dişin fizyolojik erüpsiyona devam ettiği görülmüştür. Ancak bazı durumlarda dişin pozisyonu ve çevre dokuların etkisi nedeniyle ek önlem almak gerekebilir. Bu bakımdan hastaların multidisipliner yaklaşım ile uzun dönem takibi vakanın prognozu açısından önem arz etmektedir.

NOT: Bu makale yazarlarından hiçbirinin makalede bahsi geçen konu veya malzemeyle ilgili herhangi bir ilişkisi, bağlantısı veya parasal çıkar durumu söz konusu değildir. 


\section{KAYNAKLAR}

1. Cezairli B, Taşkesen F, Coşkun Ü, Cezairli NS, Tosun E. Surgical treatment of a large complex odontoma. Meandros Med Dent J 2017;18:148.

2. Park JC, Yang JH, Jo SY, Kim BC, Lee J, Lee W. Giant complex odontoma in the posterior mandible: A case report and literature review. Imaging Sci Dent 2018;48:289-93.

3. Lehman H, Lustmann J, Regev E. Removal of an extensive mandibular odontoma using an intraoral approach. Quintessence Int 2013;44.

4. Neşe A, Yılmaz Z, Mehmet B. Süt ve daimi dişlerde sürme problemleri: 4 olgu sunumu. J Dent Fac Atatürk Uni 2018;28:233-8.

5. Iatrou I, Vardas $\mathrm{E}$, Theologie-Lygidakis $\mathrm{N}$, Leventis M. A retrospective analysis of the characteristics, treatment and follow-up of 26 odontomas in Greek children. J Oral Sci 2010;52:439-47.

6. Spini PHR, Spini TH, Servato JPS, Faria PRd, Cardoso SV, Loyola AM. Giant complex odontoma of the anterior mandible: report of case with long follow up. Braz. Dent J 2012;23:597-600.

7. Bereket C, Çakır-Özkan N, Şener İ, Bulut E, Tek M. Complex and compound odontomas: Analysis of 69 cases and a rare case of erupted compound odontoma. Niger J Clin Pract 2015;18:726-30.

8. Utumi ER, Cremonini CC, Pedron IG, Zambon CE, Cavalcanti MGP, Ceccheti MM. Maxillary reconstruction with particulate bone graft and titanium mesh: a treatment option for large complex odontoma of the maxilla. J Dent Child 2011;78:124-8.

9. Gündüz K, Büyük C. Odontoma, Adenomatoid Odontojenik Tümör. Turkiye Klinikleri J Oral and Maxillofac Radiol-Special Topics 2017;3:13-7.

10. Carvalho CHPd, Costa DdA, Queiroz LMG, Amaral JIQd, Germano AR. Extensive complex odontoma in the maxillary sinus: An uncommon presentation as a cause of chronic sinusitis. Rev Odonto Ciênc 2011;26:92-5.

11. Katiyar A, Gupta K, Solanki S, Gupta S. A case of dental hamartoma associated with unerupted primary canine in maxillary region. Nat J Maxillofac Surg 2018;9.

12. Christopher PJ, Periasamy S, Devadoss $\mathrm{P}$, Kumar SP. Surgical access to a complex composite odontoma via sagittal split osteotomy of the mandible. Cureus 2017; 9: e1915.
13. Akerzoul N, Chbicheb S, El Wady W. Suppl-1, M9: Giant complex odontoma of mandible: a spectacular case report. Open Dent J 2017;11:413.

14. Tekkesin MS, Pehlivan S, Olgac V, Aksakallı N, Alatlı C. Clinical and histopathological investigation of odontomas: review of the literature and presentation of 160 cases. J Oral Maxillofac Surg 2012;70:1358-61.

15. Raval N, Mehta D, Vachhrajani K, Nimavat A. Erupted odontoma: a case report. J Clin Diagn Res 2014; 8: ZD10-1.

16. Perumal C, Mohamed A, Singh A, Noffke C. Sequestrating giant complex odontoma: a case report and review of the literature. J Maxillofac Oral Surg 2013; 12: 480-4.

17. Agrawal B, Gharote H, Nair P, Shrivastav S. Infected complex odontoma: an unusual presentation. BMJ Case Rep 2012; 2012 2012006493.

18. Gurler G, Delilbasi C, Delilbasi E. Investigation of impacted supernumerary teeth: a cone beam computed tomograph (cbct) study. J Istanbul Univ Fac Dent 2017; 51: 18.

19. Casap N, Zeltser R, Abu-Tair J, Shteyer A. Removal of a large odontoma by sagittal split osteotomy. J Oral Maxillofac Surg 2006;64:1833-6.

20. Visioli ARC, e Silva CdO, Marson FC, Takeshita WM. Giant complex odontoma in maxillary sinus. Ann Maxillofac Surg 2015; 5: 123.

\author{
Sorumlu Yazarın Yazışma Adresi \\ Hasan Akpınar \\ Diş Hekimliği Fakültesi \\ Ağız, Diş ve Çene Cerrahisi Anabilim Dalı \\ Ordu, Türkiye \\ Telefon: 04522121283 \\ E-mail: hsnakpinar03@gmail.com
}

